\title{
Non-Hermitian Magnon-Photon Interference in an Atomic Ensemble
}

\author{
Rong Wen, ${ }^{1}$ Chang-Ling Zou, ${ }^{5}$ Xinyu Zhu, ${ }^{1}$ Peng Chen, ${ }^{1}$ Z. Y. Ou, ${ }^{4}$ J. F. Chen, ${ }^{1,6,{ }^{*}}$ and Weiping Zhang ${ }^{2,3, \dagger}$ \\ ${ }^{1}$ State Key Laboratory of Precision Spectroscopy, Quantum Institute for Light and Atoms, School of Physics and Materials Science, \\ East China Normal University, Shanghai 200241, China \\ ${ }^{2}$ School of Physics and Astronomy, Tsung-Dao Lee Institute, Shanghai Jiao Tong University, Shanghai 200240, China \\ ${ }^{3}$ Collaborative Innovation Center of Extreme Optics, Shanxi University, Taiyuan, Shanxi 030006, China \\ ${ }^{4}$ Department of Physics, Indiana University-Purdue University Indianapolis, \\ 402 North Blackford Street, Indianapolis, Indiana 46202, USA \\ ${ }^{5}$ Key Laboratory of Quantum Information, University of Science and Technology of China, Hefei 230026, Anhui, China \\ ${ }^{6}$ Shenzhen Institute for Quantum Science and Engineering and Department of Physics, \\ Southern University of Science and Technology, Shenzhen 518055, China
}

(Received 25 January 2019; published 27 June 2019)

\begin{abstract}
The interference of photons in a lossy beam splitter (BS) exhibits anticoalescence, which is surprising for bosons. Such a non-Hermitian system involving open quantum dynamics is of particular interest for quantum information processing and metrology. The Hermiticity of photonic devices is generally fixed according to the material, but is controllable at the interface of photons and atomic systems. Here, we demonstrate a tunable non-Hermitian BS for the interference between traveling photonic and localized magnonic modes. The crossover from a Hermitian to a non-Hermitian magnon-photon BS is achieved by controlling the coherent and incoherent interaction mediated by the excited levels of atoms, which is reconfigurable via the detuning of a control laser. A correlated interference pattern between the photons and magnons is demonstrated by such a non-Hermitian BS. Our system has the potential to operate with photons and magnons at the single-quanta level, and it provides a versatile quantum interface for studying the non-Hermitian quantum physics and parity-time symmetry.
\end{abstract}

DOI: 10.1103/PhysRevLett.122.253602

An optical linear beam splitter (BS) mixes the photons in different paths to interfere, and it is an essential device in various optical applications, such as the gravitational wave detection [1] and optical coherence tomography [2]. A BS can also manipulate the photonic quantum state encoded in different modes, which is the most basic device for building a quantum communication network [3-5]. Such a concept in photonics has also been generalized to other excitations, including the phonon [6], plasmon [7,8], magnon [9], and matter waves $[10,11]$. The interaction Hamiltonian for a linear BS in a most general form is

$$
\hat{H}=i \hbar\left(g \hat{b} \hat{c}^{\dagger}-g^{*} \hat{b}^{\dagger} \hat{c}\right) e^{i \delta \phi},
$$

where $g$ is a complex coefficient coupling the modes $b$ and $c$. Most of the previous studies considered a closed system and the extra phase $\delta \phi$ is simply 0 or $\pi$ to make the Hamiltonian a Hermitian. However, practical quantum systems are open to the environment and $e^{i \delta \phi}$ takes other values to transform the system into a non-Hermitian one. Previous works demonstrated that loss in a non-Hermitian system gives rise to a nonunitary transformation [12], and the quantum operations on photons would be affected in such a non-Hermitian system [13]. For example, anticoalescence, i.e., each particle occupying an individual output port, has been demonstrated for interfering bosons in such a nonunitary BS [14]. Two-photon absorption [15], i.e., either both or no photons are absorbed, is applicable to create a quantum gate to control absorption with a lossy linear BS.

While the interference in non-Hermitian system is of great importance conceptually and fundamentally, realizing a fully controllable non-Hermitian BS, i.e., with tunable $e^{i \delta \phi}$, is still a challenge. Non-Hermitian physics and parity-time symmetry [16-19] in atomic systems [20,21] are attracting more and more attention for their flexibility. Coherent conversion processes between atomic excitations and optical photons could be treated as linear BS interactions [22-24], in which the atomic and optical modes are distinguished channels. A hybrid atom-light interface provides an appealing and versatile platform [25-27] to explore the applications in quantum storage [28-30], entangling distant quantum nodes $[22,31]$ and interferometers $[32,33]$.

Driven by an external optical field, magnons in an atomic ensemble, the quasiparticle of collective atomic spin-wave excitation [34-38], could be converted to photons with dissipation presented at the interface. The dissipation in such a BS model does not only lead to a change of photon number, but significantly alters the unitary transformation. In this Letter, we report that an ensemble of three-level atoms driven by a near-resonant control field constitutes a 

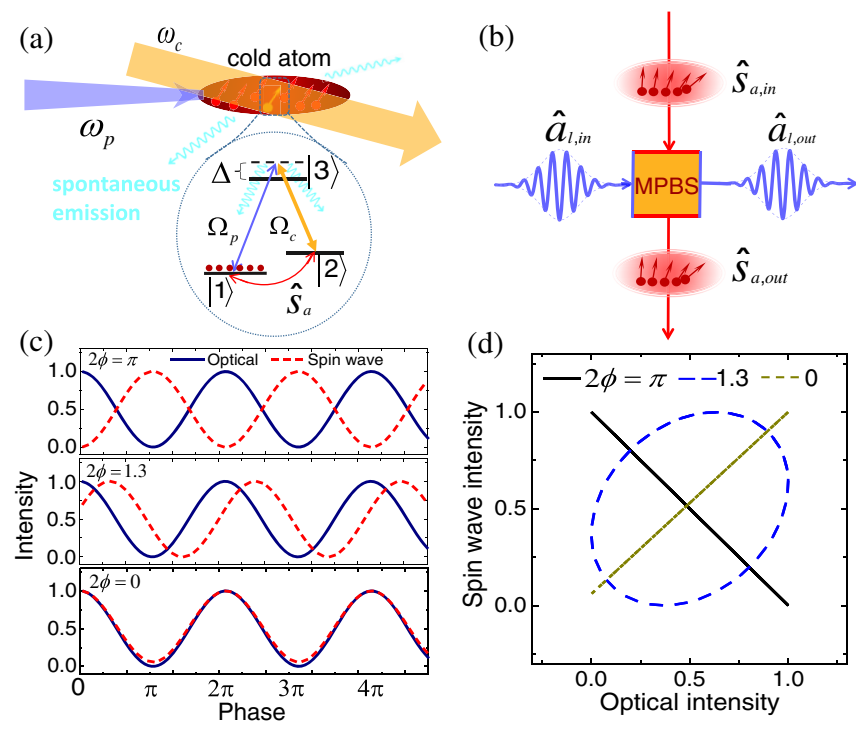

FIG. 1. Schematics of the non-Hermitian MPBS. (a) Magnonphoton conversion in an ensemble of three-level atoms, where a control beam can stimulate the coherent conversion between the input probe photons and collective atomic excitation between the two metastable states $|1\rangle$ and $|2\rangle$ (magnon), while the spontaneous emission induces an incoherent conversion between photon and magnon. The inset: three-level $\Lambda$ atomic energy level. Ground state $|1\rangle(|2\rangle): 5 S_{1 / 2}, F=2(F=3)$; excited state $|3\rangle: 5 P_{1 / 2}, F=3$. The frequencies of probe and control beams satisfy the two-photon resonant condition. (b) The cold atom ensemble serves as a beam splitter, where the input optical wave and spin wave interfere. (c) The sinusoidal fringes of interference between the optical wave and spin wave. (d) The phase diagram for the photon and magnon output, with the black solid and green dot lines presenting the phase difference of fringe $2 \phi=\pi$ and 0 , respectively. The ellipse represents an arbitrary non-Hermitian beam splitter.

tunable non-Hermitian magnon-photon beam splitter (MPBS). The efficient tuning knob for Hermiticity is the single-photon detuning in the electromagnetically induced transparency (EIT) configuration [39]. The whole process of interference between spin-wave excitations and light constitutes a temporal Mach-Zehnder (MZ) interferometer, in which the MPBS reveals interesting unconventional phenomena of the non-Hermitian interface. It is worth noting that our experiments are performed with classical coherent states, while the principle of the reconfigurable non-Hermitian BS is valid for single excitation level. The new mechanism reported in this work will provide a new perspective on quantum state transfer between atom and light, and finds its potential application in quantum information processing and quantum network.

As sketched in Fig. 1(a), the non-Hermitian MPBS is realized by a control laser driving an atomic ensemble and stimulating conversion between the signal photons and magnons. The underlying quantum processes is explained by the energy level diagram [inset of Fig. 1(a)]: a strong control laser $\left(\Omega_{c}\right)$ induces coherent transition between $|2\rangle$ and $|3\rangle$, and also initializes the atomic ensemble in the ground state $|1\rangle$; signal photons $\left(\Omega_{p}\right)$ can be converted to magnons, i.e., the excitation coherence between levels $|1\rangle$ and $|2\rangle$, through the intermediate excited state $|3\rangle$. Because of the spontaneous emission of atomic excited state, there are two incoherent processes associated with the MPBS operation: (i) By directly exciting atoms to $|3\rangle$, the signal photon be absorbed and scattered to free space; (ii) the population on $|2\rangle$ be excited to $|3\rangle$ by the control laser, and decay to $|1\rangle$ by emitting a photon into free space. Although the two processes would lead to the loss of photon and magnon separately, they are not independent because both processes couple with the same excited state and free-space optical modes. Therefore, the two decoherence processes would interfere on the shared decay channel, allowing an indirect magnon-photon conversion process mediated by the decay channels; i.e., the signal couples to the free-space mode and sequentially couples to the magnon mode, or vice versa. As a result, the spontaneous emission of excited state contributes an effective incoherent interaction between magnon and photon, which laid as the foundation of the non-Hermitian MPBS studied in this work.

Figure 1(b) conceptually illustrates the MPBS: a traveling optical signal mode $\left(\hat{a}_{l}\right)$ and a localized magnon mode $\left(\hat{S}_{a}\right)$ are mixed via the MPBS. The magnons are prepared through converting part of the signal optical field into the atomic excitations and are then set with different initial phase $\theta$. They again interfere with the other part of the signal optical field on the MPBS. The whole process composes a MZ interferometer, and the intensity studies of output photons and magnons would show sinusoidal interference fringes with the interferometric phase $\theta$. To investigate the Hermiticity of the MPBS, we introduce a phase factor $\phi$ for the reflection coefficients $\left(r=|r| e^{i \phi}\right.$, $\left.r^{\prime}=\left|r^{\prime}\right| e^{i \phi}\right)$, with transmission coefficients $\left(t, t^{\prime}\right)$ to be real. Take an ideal unitary BS as an example, $\phi=\pi / 2$, i.e., $t= \pm i r$ with $|t|=|r|$ for simplicity. In this case, the interference between photon and magnon for different $\theta$ show complementary oscillations due to the energy conservation, as shown in Fig. 1(c), in which the phase shift between fringes is $2 \phi=\pi$. In contrast, for a nonunitary BS with $\phi \neq \pi / 2$ [40], the interference fringes for two ports are not complementary to each other as shown in the lower row in Fig. 1(c). By plotting the Lissajous curve of the outputs from two ports as Fig. 1(d), the output intensities between the magnonic and optical modes can indicate anticorrelation $(2 \phi=\pi)$, with Hermitian interaction. By controlling the MPBS, the output modes deviate from anticorrelation, exhibiting a non-Hermitian system, and finally become correlation $(2 \phi=0, t= \pm r)$.

The experimental setup and schematics are shown in Fig. 2. A cigarette-shaped laser cooled ${ }^{85} \mathrm{Rb}$ atom cloud is prepared in a two-dimensional magneto-optical trap (MOT) $[41,42]$. We use a three-level EIT setup $[25,43]$ to perform the MPBS. The probe and coupling lasers, which are 
(a)
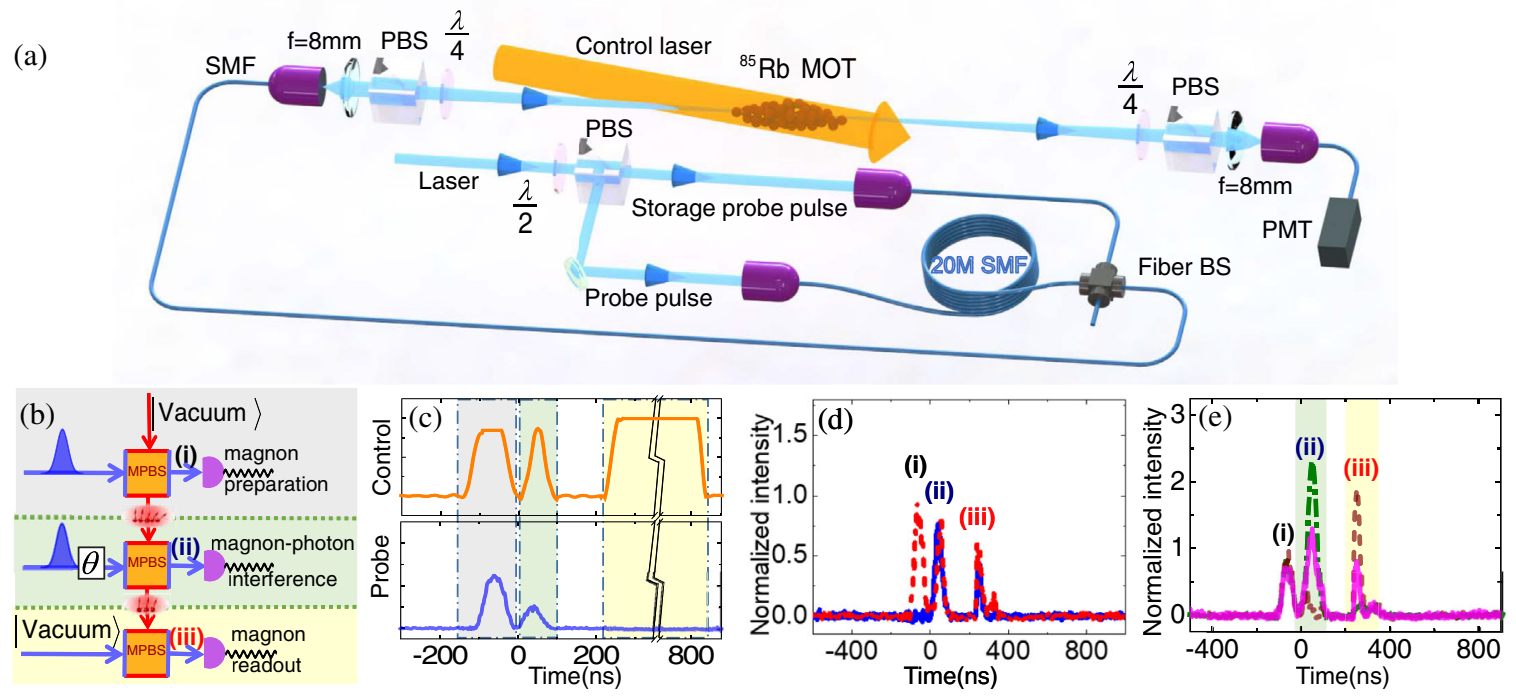

FIG. 2. Experimental setup. (a) The weak probe pulse splitting into two pulses are launched into an ${ }^{85}$ Rb MOT (with a 20-meter-fiber inserted) along the longitudinal axis of the atomic cloud; the angle between the control beam and the atom long axis direction is $2.5^{\circ}$. The output for the photon is directly collected by a PMT. (b) Schematic diagram of the magnon-photon interference. (c) The timing sequence of the control light and the probe light is represented by the orange and the blue lines. (d) The output signals of MPBS with only an optical signal (blue solid line), and with only spin-wave excitation (red dash line) are presented. (e) Magnon-photon interference at different interferometric phase 0 (the brown dashed line), $\pi / 2$ (the magenta solid line), and $\pi$ (the green dot-dashed line), when both the optical pulse and spin wave exist, with $\Delta=30 \mathrm{MHz}$ and an OD of 30. The MPBS output intensities of the photon and magnon can hence be obtained by integrating the areas of the output pulses [the green and yellow region in (e)]. In (d),(e), the optical intensity is normalized so that the peak of the transmitted pulse denoted as (i) is 1, while the typical average photon number in the pulse (ii) or (iii) is 1000 .

originated from the same diode laser, are, respectively, frequency shifted using acousto-optic modulators (AOM). The control laser is blue detuned from the transition $|2\rangle \leftrightarrow$ $|3\rangle$ by $\Delta$, and the probe is blue detuned from the transition $|1\rangle \leftrightarrow|3\rangle$ to maintain two-photon resonance. The two weak probe pulses [gray and pale green shadows in Fig. 2(c)] are generated by a single $50 \mathrm{~ns}$ pulse that split by an optical beam splitter, with their time separation of around $100 \mathrm{~ns}$. The magnons are prepared via storing collective atomic spin-wave excitations through the first weak probe pulse sent to the atomic cloud (being driven by the first control pulse), which is equivalent to a quantum storage process [44]. To realize the temporal MZ interferometer by the MPBS, the delayed probe pulse is sent into the atom cloud after the magnon preparation. By a second control pulse, the optical output of the interferometer is directly collected by a photomultiplier tube (PMT). Sequentially, the magnons in the atomic ensemble are read out by converting to optical signals, which is also detected by the PMT. Therefore, a long control pulse with a duration of $600 \mathrm{~ns}$ [yellow shadows in Figs. 2(b), 2(c), and 2(e)] is applied to the atomic ensemble after each cycle of the interference experiments. It is used for magnon readout, and also for depleting the residue magnons in an atomic cloud. Since our practical experimental time (about $1 \mu \mathrm{s}$ ) is very short compared with the cycling time $(5 \mathrm{~ms})$ and the phase uncertainty of the fiber is negligible (according to
Ref. [45], the typical fiber phase drift during a single experiment cycle is smaller than $18 \mathrm{rad} / \mathrm{ms} / 20 \mathrm{~km} \times$ $20 \mathrm{~m} \times 1 \mu \mathrm{s} \sim 1.8 \times 10^{-6} \mathrm{rad}$ ), the relative phase of the probe pulses is not controlled manually but fluctuates randomly. Therefore, we record the output intensities of photon and magnon in the phase diagram, where the intensities are obtained by integrating the areas of the output pulses. Figure 2(d) shows the outputs of the MPBS with input at one port, and Fig. 2(e) shows the typical output results of the MPBS interference.

For a simplified model of our experiments based on the single magnon mode, the MPBS operation can be described by the effective Hamiltonian $(\hbar=1)$ [22]

$$
\begin{aligned}
\hat{H}_{\mathrm{eff}}= & \frac{g^{2} N}{\Delta-i \gamma_{13}} \hat{a}_{l}^{\dagger} \hat{a}_{l}+\frac{\Omega_{c}^{2}}{\Delta-i \gamma_{13}} \hat{S}_{a}^{\dagger} \hat{S}_{a} \\
& +\frac{g \sqrt{N} \Omega_{c}}{\Delta-i \gamma_{13}}\left(\hat{a}_{l} \hat{S}_{a}^{\dagger}-\hat{a}_{l}^{\dagger} \hat{S}_{a}\right),
\end{aligned}
$$

under the approximations of $|\Delta|^{2}+\gamma_{13}^{2} \gg g, \Omega_{c} . \hat{a}_{l}$, and $\hat{S}_{a}$, respectively, denotes the annihilation operator for photons and magnons, both of which are bosons. Here, $\Delta=\omega_{3}-\omega_{1}-\omega_{p}$ is the detuning, $\gamma_{13}$ represents the dephasing rate between $|3\rangle$ and $|1\rangle, g$ is the atom-photon coupling strength, $N$ is the number of atoms, and $\Omega_{c}$ is the Rabi frequency of the control field. In all experiments, the signal and control lasers satisfy the two-photon resonance 
condition, i.e., $\omega_{p}-\omega_{c}=\omega_{2}-\omega_{1}$. The last term of Eq. (1) denotes the linear beam splitter type of interaction [22], but with a complex coupling strength considering loss, which actually works as the tunable extra factor $e^{i \delta \phi}$. The nonzero imaginary part leads to the non-Hermitian [19] magnonphoton conversion in the cold atom ensemble. $\hat{a}_{l}$ evolves with the $z$ position along the longitudinal axis of the atomic cloud, and therefore we denote $\hat{A}_{\text {in }}$ and $\hat{A}_{\text {out }}$ for the photons at position $z=0$ and $L$, respectively. $\hat{S}_{a}$ evolves with the interaction time $\tau$, with $\hat{M}_{\text {in }}$ and $\hat{M}_{\text {out }}$ for magnons at $\tau=0$ and $\tau_{p}$ (considering the signal pulse is a square pulse with duration of $\tau_{p}$ ). The spatial-temporal input-output relation of the non-Hermitian MPBS can be represented by $\left(\begin{array}{c}\hat{M}_{\text {out }} \\ \hat{A}_{\text {out }}\end{array}\right)=T\left(\begin{array}{c}\hat{M}_{\text {in }} \\ \hat{A}_{\text {in }}\end{array}\right)$, where the transfer matrix is [43]

$$
T=\left(\begin{array}{cc}
\xi & -(1-\xi) \\
-\frac{\eta}{\zeta}(1-\xi) & 1-\frac{\eta}{\zeta}(1-\xi)
\end{array}\right) .
$$

The phase difference between two interference fringes is then

$$
2 \phi=\arg [1-1 / \xi]+\arg \left(\frac{-\frac{\eta}{\zeta}(1-\xi)}{1-\frac{\eta}{\zeta}(1-\xi)}\right) .
$$

Here, $\xi=e^{-\left\{[\zeta] /\left[\left(i \Delta / \gamma_{13}+1\right)\right]\right\}} . \zeta=\Omega_{c}^{2} \tau_{p} / \gamma_{13}$ is a dimensionless number quantify the beam splitter interaction strength, and normally $\zeta>1 . \eta$ denotes a parameter proportional to the optical depth (OD).

From the transfer matrix, the parameter $\Delta / \gamma_{13}$ plays an important role to control the loss and hence the boson coalescence within MPBS. With far-detuned control, i.e., $\Delta / \gamma_{13} \gg 1, T_{11} \approx 1+i \zeta \gamma_{13} / \Delta, T_{22} \approx 1+i \eta \gamma_{13} / \Delta, T_{12} \approx$ $i \zeta \gamma_{13} / \Delta$ and $T_{21} \approx i \eta \gamma_{13} / \Delta$, the MPBS therefore behaves as a normal lossless BS with $\phi \approx \pi / 2$. The phase difference of the interference fringe is $\pi$, as shown by our experimental results in Fig. 3(a). The output intensity of the optical port reaches the maximum while the atomic magnon port reaches the minimum, and vice versa, showing anticorrelation that conserves the number of excitations against $\theta$. Oppositely, under near-resonance control $\Delta / \gamma_{13} \rightarrow 0$, the MPBS is a lossy BS with off-diagonal terms of $T$ as negative numbers, i.e., $T_{12(21)} \approx-T_{11(22)}$. The outputs are correlated and tend to simultaneously reach extreme values, and the phase difference of fringes is zero, as shown in Fig. 3(e). An implication of this result is that it is in the PTsymmetry broken regime of the non-Hermitian system $[13,19]$. There is only one eigenvalue for the mode of photon and magnon, so the ratio of the output at two ports is invariant against $\theta$. For a general non-Hermitian BS with $0<2 \phi<\pi$, the MPBS output shows an ellipse trajectory.

As indicated by Eq. (2), the phase difference of interference fringes $2 \phi$, i.e., the non-Hermitianity, can be altered by controlling the experiment parameters of the MPBS, including the detuning $\Delta$ and OD. The phase $\phi$ of MPBS can be obtained by reconstructing the Lissajous curve. In
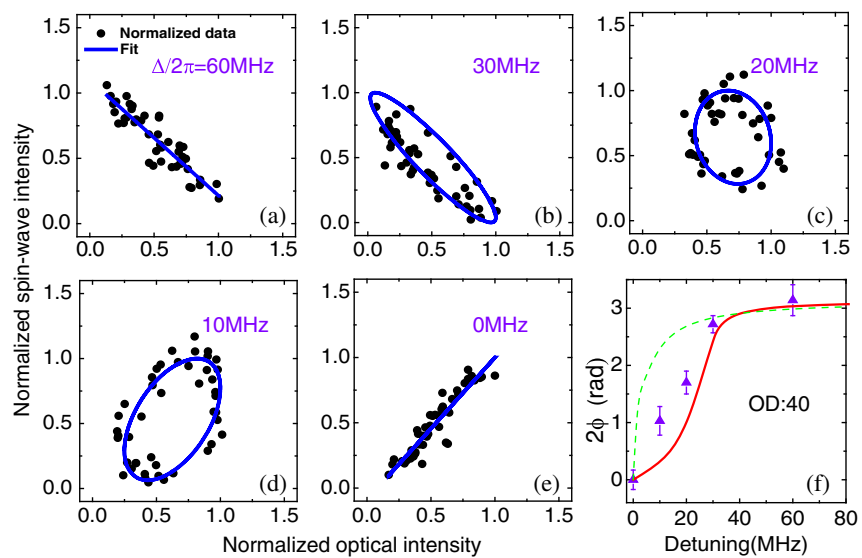

FIG. 3. The transition from Hermitian to non-Hermitian MPBS. The black dots represent the experimental data, and the blue solid lines show the fitting ellipse curves. (a)-(e) The phase diagram with an OD of 40 and with different single photon detunings $\Delta$. (f) $2 \phi$ vs $\Delta$. The green dashed line denotes analytical results from Eq. (3), and the red solid line is plotted according to the numerical simulation of the magnon-photon coupling model described in the Supplemental Material [43]. The error bars are plot according to all the variation of the fit ellipses covering the data.

Figs. 3(a)-3(e), we decrease single photon detuning $\Delta / 2 \pi$ from $60 \mathrm{MHz}$ to 0 while maintaining $\mathrm{OD}$ as 40 . The experimental data are fitted with blue solid ellipses, which change from anti correlated to correlated with $\Delta / \gamma_{13}$ decreases. As summarized in Fig. 3(f), with theoretical lines calculated from the full magnon-photon coupling model [43], the single mode approximation satisfactorily describes the transition from Hermitian to non-Hermitian. Figures 4(a)-4(c) represent the measured phase diagram for
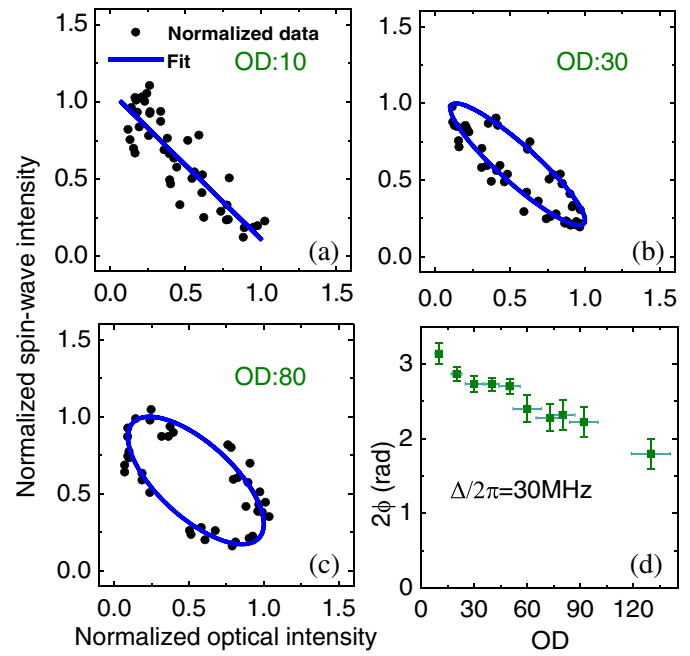

FIG. 4. The control of non-Hermitian magnon-photon interference through OD. (a)-(c) The phase diagram with single photon detuning $\Delta / 2 \pi=30 \mathrm{MHz}$ with different ODs. The black dots represent the experimental data, and the blue solid lines show the fitting ellipse curves. (d) $2 \phi$ vs OD. The error bars are plot according to all the variation of the fit ellipse covering the data. 
different ODs, with fixed $\Delta / 2 \pi=30 \mathrm{MHz}$, and they are summarized in Fig. 4(d). For increasing OD, the number of atoms $N \propto$ OD increases, where the single mode approximation breaks down. It can be intuitively understood that a MPBS with increased OD can be treated as a sequence of spatially cascaded single magnon mode-based MPBS; thus the non-Hermitian induced phase would accumulate with increasing the OD. From Fig. 4(d), it is clearly demonstrated that the MPBS deviated from Hermitian; i.e., $2 \phi$ decreases from $\pi$, for growing OD. Thus, this MPBS can be easily reconfigured from a nearly ideal Hermitian model to a non-Hermitian beam splitter by only changing the laser frequencies or the condition of the MOT.

In summary, a reconfigurable non-Hermitian magnonphoton conversion is demonstrated in an atomic ensemble, with the assistance of a control laser in the EIT configuration. The non-Hermitian coupling originates from the open system dynamics of the system, where the spontaneous emission of the excited state to the free-space photonic channels contribute to the nontrivial indirect coupling between the coherent magnonic and photonic excitations, which eventually give rise to the nonunitary beam splitter behavior. By adjusting the control laser detuning $\Delta$ to be large enough, the excited state of the atoms is rarely excited and thus the coupling between the coherent excitations to the free-space decay channels are suppressed, and the beam splitter is close to unitary. In contrast, the beam splitter shows significant non-Hermitian features when the control laser is near resonant with the atoms. The interface between atoms and light we demonstrated here can be extended to all types of atomic ensembles and atomlike systems. A single quanta injection of photon and magnon is plausible in our proposed atomic system. A direct implication is the quantum statistics of bosons can be instantly changed in such exotic BS system. This is caused by the non-unitary two-mode transformation induced by an incoherent magnon-photon interaction. There is potential for developing a nonlinear quantum gate in a linear system via controlling loss [15]. Further, by exploiting the multiple level atoms and optical control, our results can be generalized to multiple magnonic modes and photons with different colors, and even to bilinear-type interactions for studying the interplay between gain [46] and loss.

This work is supported by the National Key Research and Development Program of China under Grant No. 2016YFA0302001, and the National Natural Science Foundation of China through Grants No. 11674100, No. 11654005, No. 11234003, and No. 91436211, Quantum Information Technology, Shanghai Science and Technology Major Project, the Natural Science Foundation of Shanghai No. 16ZR1448200, and Shanghai Rising-Star Program No. 17QA1401300. C.-L.Z. is supported by Anhui Initiative in Quantum Information Technologies (AHY130200) and National Natural Science Foundation of China Grant No. 11874342. *jfchen@phy.ecnu.edu.cn wpz@sjtu.edu.cn

[1] B. P. Abbott, Phys. Rev. Lett. 116, 061102 (2016).

[2] A. I. Lvovsky and M. G. Raymer, Rev. Mod. Phys. 81, 299 (2009).

[3] P. Hariharan, Rep. Prog. Phys. 54, 339 (1991).

[4] M. Zukowski, A. Zeilinger, M. A. Horne, and A. K. Ekert, Phys. Rev. Lett. 71, 4287 (1993).

[5] P. Walther, J. W. Pan, M. Aspelmeyer, R. Ursin, S. Gasparoni, and A. Zeilinger, Nature (London) 429, 158 (2004).

[6] K. Toyoda, R. Hiji, A. Noguchi, and S. Urabe, Nature (London) 527, 74 (2015).

[7] J. S. Fakonas, H. Lee, Y. A. Kelaita, and H. A. Atwater, Nat. Photonics 8, 317 (2014).

[8] R. W. Heeres, L. P. Kouwenhoven, and V. Zwiller, Nat. Nanotechnol. 8, 719 (2013).

[9] A. V. Chumak, V. I. Vasyuchka, A. A. Serga, and B. Hillebrands, Nat. Phys. 11, 453 (2015).

[10] A. Lenef, T. D. Hammond, E. T. Smith, M. S. Chapman, R. A. Rubenstein, and D. E. Pritchard, Phys. Rev. Lett. 78, 760 (1997).

[11] R. Lopes, A. Imanaliev, A. Aspect, M. Cheneau, D. Boiron, and C. I. Westbrook, Nature (London) 520, 66 (2015).

[12] S. M. Barnett, J. Jeffers, A. Gatti, and R. Loudon, Phys. Rev. A 57, 2134 (1998).

[13] M. Li, C. Zou, G. Guo, and X. Ren, Chin. Optic. Lett. 15, 092701 (2017).

[14] B. Vest, M. C. Dheur, É. Devaux, A. Baron, E. Rousseau, J. P. Hugonin, J. J. Greffet, G. Messin, and F. Marquier, Science 356, 1373 (2017).

[15] T. Roger, S. Restuccia, A. Lyons, D. Giovannini, J. Romero, J. Jeffers, M. Padgett, and D. Faccio, Phys. Rev. Lett. 117, 023601 (2016).

[16] Z. P. Liu, J. Zhang, Ş. K. Özdemir, B. Peng, H. Jing, X. Y. Lü, C. W. Li, L. Yang, F. Nori, and Y. X. Liu, Phys. Rev. Lett. 117, 110802 (2016).

[17] W. Chen, Ş. K. Özdemir, G. Zhao, J. Wiersig, and L. Yang, Nature (London) 548, 192 (2017).

[18] C. M. Bender and S. Boettcher, Phys. Rev. Lett. 80, 5243 (1998).

[19] R. El-Ganainy, K. G. Makris, M. Khajavikhan, Z. H. Musslimani, S. Rotter, and D. N. Christodoulides, Nat. Phys. 14, 11 (2018).

[20] Z. Zhang, Y. Zhang, J. Sheng, L. Yang, M. A. Miri, D. N. Christodoulides, B. He, Y. Zhang, and M. Xiao, Phys. Rev. Lett. 117, 123601 (2016).

[21] P. Peng, W. Cao, C. Shen, W. Qu, J. Wen, L. Jiang, and Y. Xiao, Nat. Phys. 12, 1139 (2016).

[22] K. Hammerer, A. S. Sørensen, and E. S. Polzik, Rev. Mod. Phys. 82, 1041 (2010).

[23] Z. Y. Ou, Phys. Rev. A 78, 023819 (2008).

[24] K. F. Reim, J. Nunn, X. M. Jin, P. S. Michelberger, T. F. M. Champion, D. G. England, K. C. Lee, W. S. Kolthammer, N. K. Langford, and I. A. Walmsley, Phys. Rev. Lett. 108, 263602 (2012).

[25] X. Zhu, R. Wen, and J. F. Chen, Phys. Rev. A 97, 063801 (2018).

[26] W. Kozlowski, S. F. Caballero-Benitez, and I. B. Mekhov, Phys. Rev. A 94, 012123 (2016). 
[27] G. E. Marti, A. MacRae, R. Olf, S. Lourette, F. Fang, and D. M. Stamper-Kurn, Phys. Rev. Lett. 113, 155302 (2014).

[28] M. Fleischhauer and M. D. Lukin, Phys. Rev. Lett. 84, 5094 (2000).

[29] D. F. Phillips, A. Fleischhauer, A. Mair, R. L. Walsworth, and M. D. Lukin, Phys. Rev. Lett. 86, 783 (2001).

[30] Y. Wang, J. Li, S. Zhang, K. Su, Y. Zhou, K. Liao, S. Du, H. Yan, and S.-L. Zhu, Nat. Photonics 13, 346 (2019).

[31] D. N. Matsukevich and A. Kuzmich, Science 306, 663 (2004).

[32] C. Qiu, S. Chen, L. Q. Chen, B. Chen, J. Guo, and W. Zhang, Optica 3, 775 (2016).

[33] G. Campbell, M. Hosseini, B. M. Sparkes, P. K. Lam, and B. C. Buchler, New J. Phys. 14, 033022 (2012).

[34] L. M. Duan, M. D. Lukin, J. I. Cirac, and P. Zoller, Nature (London) 414, 413 (2001).

[35] A. V. Gorshkov, A. Andre, M. Fleischhauer, A. S. Sørensen, and M. D. Lukin, Phys. Rev. Lett. 98, 123601 (2007).

[36] R. Zhao, Y. O. Dudin, S. D. Jenkins, C. J. Campbell, D. N. Matsukevich, T. A. B. Kennedy, and A. Kuzmich, Nat. Phys. 5, 100 (2009).
[37] S.-J. Yang, X.-J. Wang, J. Li, J. Rui, X.-H. Bao, and J.-W. Pan, Phys. Rev. Lett. 114, 210501 (2015).

[38] B. Chen, C. Qiu, S. Chen, J. Guo, L. Q. Chen, Z. Y. Ou, and W. Zhang, Phys. Rev. Lett. 115, 043602 (2015).

[39] S. E. Harris, Phys. Today 50, No. 7, 36 (1997).

[40] A. Zeilinger, Am. J. Phys. 49, 882 (1981).

[41] J. Zhang, Z. J. Gu, P. Qian, Z. G. Han, and J. F. Chen, Chin. Phys. Lett. 32, 064211 (2015).

[42] S. Zhang, J. F. Chen, C. Liu, S. Zhou, M. M. T. Loy, G. K. Wong, and S. Du, Rev. Sci. Instrum., 83, 073102 (2012).

[43] See Supplemental Material at http://link.aps.org/ supplemental/10.1103/PhysRevLett.122.253602 for a detailed derivation.

[44] Y.-F. Hsiao, P.-J. Tsai, H.-S. Chen, S.-X. Lin, C.-C. Hung, C.-H. Lee, Y.-H. Chen, Y.-F. Chen, I. A. Yu, and Y.-C. Chen, Phys. Rev. Lett. 120, 183602 (2018).

[45] M. Lucamarini, Z. L. Yuan, J. F. Dynes, and A. J. Shields, Nature (London) 557, 400 (2018).

[46] F. Hudelist, J. Kong, C. Liu, J. Jing, Z. Y. Ou, and W. Zhang, Nat. Commun. 5, 3049 (2014). 